\title{
Adapting to Different Needs in Different Locations: Handheld Com- puters in University Education
}

\author{
Susan Bull, Yanchun Cui, Harald Röbig and Mike Sharples \\ Electronic, Electrical and Computer Engineering, University of Birmingham, U.K. \\ s.bull@bham.ac.uk
}

\begin{abstract}
Educational use of handheld computers is becoming more common. It is therefore important to examine differences between standard educational interactions, and possibilities offered by handheld devices. Handheld computers allow the opportunity to study at times and locations where individualised interactions would not normally be possible or convenient. Conditions in such locations may differently affect a user's choice of task or ability to carry out an activity successfully. This paper introduces two adaptive systems: (1) a system that adapts to learner knowledge as in a standard intelligent tutoring system, but also to time available for study and location-related features of concentration level and likelihood of interruption; (2) a system providing easy access to commonly used files, applications and tasks, according to location of use.
\end{abstract}

\section{Introduction}

With increasing adoption of mobile technology in education it is important to examine appropriate uses for this new technology, with reference to the context in which it is used. Work on adapting interactions to a learner's location, providing information relevant to that location, is in line with Dey and Abowd's definition of context-awareness as: "the use of context to provide task-relevant information and/or services to a user, wherever they may be" [1]. A common example is visitors to museums or art galleries. Zancanaro et al's museum guide uses infrared sensors to detect a user's location, then presents relevant multimedia information according to the fresco painting the user is currently viewing [2]. Zimmermann et al offer audio presentations to a visitor to an art exhibition according to their location, but also take into account a profile of the user, to better individualise presentations [3]. The context awareness subsystem of MOBIlearn provides content and services to users based on attributes of the setting (location, available artefacts, online partners) and the user (user profile, time spent at current location) [4]. There are a few systems for handheld computers that model a user's understanding, as in traditional intelligent tutoring systems (ITS). Ketamo's Adaptive Geometry Game adapts questions based on a child's accuracy and speed of response to multiple choice questions [5]. Bull and McEvoy's C-POLMILE adapts material according to a university student's answers to multiple choice questions in $\mathrm{C}$ programming [6]. Interactions with these systems are not location-dependent.

An area that has received less attention is that of modelling both understanding and features of location that affect a user's ability to learn, e.g. level of concentration in a location, and likelihood of interruption by others. Thus, unlike the previous examples, the location-relevant information does not determine content of an interaction, but rather, the type of interaction most suitable under the current circumstances. This is a key feature of handheld computers that is often less relevant to the desktop PC. A second issue is the amount of time learners have, as they may be more likely to use a handheld device for brief periods. We introduce TenseITS, an ITS for handheld computers that adapts to knowledge as in a traditional ITS, but that also structures interactions appropriately for the user in their current location, considering their ability to concentrate and the likelihood of interruption in that location, and the time they have available for study.

Another area relevant to everyday activities is student use of files, task lists and applications. Individual, but consistent usage patterns were found for university students using handheld computers in different locations [7], suggesting an adaptive interface to facilitate access to files, etc., depending on location. We present My Chameleon, designed according to these findings.

TenseITS and My Chameleon are intended for integration into a student's daily routine.

\section{Adapting to Location}

TenseITS: Mobile learners should be enabled to learn at times and locations of their choice [8], and issues such as concentration and possibility of interruption may vary in different locations, so may be usefully modelled $[9,10]$. TenseITS enables the mobile learner to take advantage of individualised learning opportuni- 
ties that would not otherwise be possible, fitting around their daily routine without disrupting other activities. It is intended primarily for Chinese learners of advanced English (e.g. students at an English-speaking university); the domain of tenses chosen as many students find tense challenging, as time is expressed differently in Chinese [11]. (See [10] for language issues relating to TenseITS.) Learner attributes modelled are:

- knowledge - inferred from correct responses to multiple choice questions

- misconceptions - inferred from incorrect responses matched with items in a misconceptions library

- general difficulties - indicated by incorrect responses that do not suggest misconceptions

Multiple choice questions are used due to the ease with which responses can be made using a handheld device.

TenseITS needs to know the user's learning context, thus also modelling:

- location - entered explicitly by the learner

The user can add locations to the default list. As it is the type of location (e.g. library, restaurant, 'travelling'), and the way this kind of location affects the individual user that is important, rather than the actual position, GPS positioning is not used. Similarly, the user's calendar is not used to infer location for two reasons: (1) students often do not stick to their schedule as observed by their absence from lectures! (2) TenseITS is designed for the short periods between other activities - such times are often not entered into the calendar. The following features of location that may affect the user's ability to focus, are modelled:

- concentration (high, medium, low)

- likelihood of interruption (high, medium, low)

These are initially input by the user. Once there is an entry, TenseITS infers the most likely levels of concentration and interruption on subsequent uses in that location, and offers the most commonly chosen as default selections. Also modelled:

- available time - input by the user

The student explicitly provides information on length of time. Again, manual input is necessary because time between activities is not usually explicitly scheduled. The main timespans offered are: less than 15 minutes, and 15-30 minutes. (Investigation of how accurately time is judged may enable refinement of these choices.) Also offered: 30-60 minutes, and 60 minutes plus, in case users may sometimes wish to use TenseITS for longer periods (e.g. on a long train journey).

The method of obtaining information is shown in Figure 1, where the user selects location and amount of time, and can change inferred values for concentration and interruption. Comparing time, concentration, likelihood of interruption to the learner model attributes (knowledge, difficulties, misconceptions), TenseITS offers an interaction appropriate for the location, time and current learning needs - e.g. in a situation where a learner is likely to be frequently interrupted, the interaction will not require continued focus. Depending on learner needs, this may be revision of a previous topic or questions on a current topic. Where the learner can concentrate and is unlikely to be interrupted, they may get new material (but revision material or questioning will be recommended if the learner model suggests this is currently more useful). If the user has enough time, a combination of activities may be offered (e.g. tutorial followed by questions, feedback). The examples of interactions in Figure 1 are: tutorial; questions; feedback.
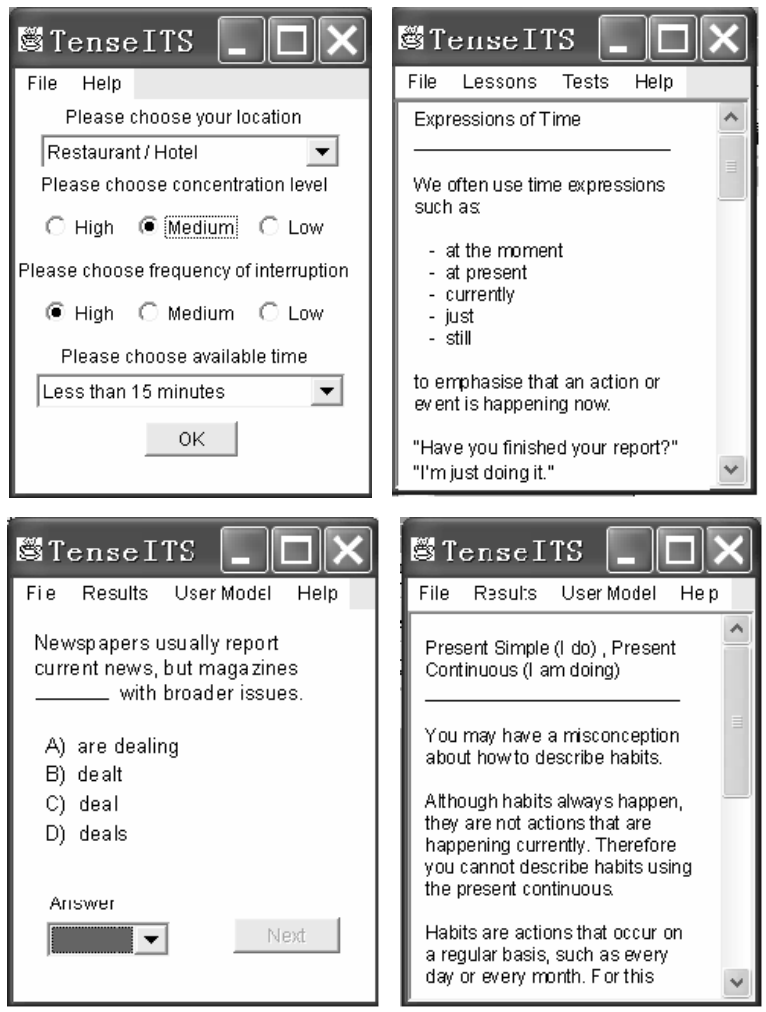

Figure 1. TenselTS - Screens

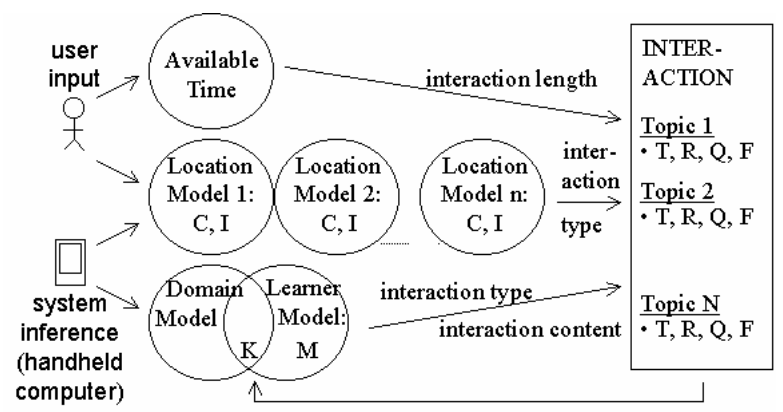

Figure 2. TenselTS - Interaction Key: C - concentration; I - interruption; $\mathrm{K}$ - knowledge; $\mathrm{M}$ - misconceptions; T - tutorial; $\mathrm{R}$ - revision; $\mathrm{Q}$ - questions; F - feedback 
The feedback takes the user's understanding, including misconceptions, as a starting point, to facilitate consciousness-raising (argued to be important in language learning [12]). The example in Figure 1 explains why the learner should not use the tense they have been using, before continuing with an explanation.

Figure 2 shows that the learner provides information about their location and features of the location relevant to their ability to learn in that location; and the amount of time they have. The time model is relevant for a single session, containing only information about the length of the session. There are multiple location models, each holding information about the user's typical concentration and likelihood of interruption in that location. These values are inferred from explicit input of the user over time, as shown in Figure 1, and are used unless the user overrides them. The learner model (knowledge, difficulties, misconceptions) is inferred and updated according to the student's answers to multiple choice questions. The time model allows TenseITS to determine the appropriate length of an interaction (how much content, how many questions, whether to combine different interaction types). The location model enables selection of an appropriate interaction type (tutorial, revision, questions, feedback). The learner model also allows inference of appropriate interaction types, but in relation to current knowledge state, rather than ability to study well in a particular location. The learner model also, as in all ITSs, allows selection of appropriate content. These location and knowledge attributes are combined to make the final selection. For example, a user on a 15 minute bus journey who is unlikely to be interrupted, but finds it difficult to concentrate because they get distracted (e.g. looking out the window), may receive revision questions even if there is a suitable new topic to introduce according to the learner model. This is because the learner can easily switch between answering questions and looking out the window without having to review parts of the interaction before continuing. However, the same learner in the library, having finished an assignment with 15 minutes left before the next lecture, may be given a short introduction to a new topic as in this location their concentration is high, and the likelihood of interruption is low.

My Chameleon: My Chameleon also supports differences in user needs in different locations. This is a much simpler approach, providing an adaptive interface to enable easy access to the files, applications and tasks commonly used by a student in a variety of locations. The attributes modelled are, for example, simply:

- frequency of access: file X location A; location B

- frequency of access: file Y location A; location B

- frequency of access: application X loc. A; loc. B

- frequency of access: application Y loc. A; loc. B

- frequency of access: task X location A; location B
- frequency of access: task Y location A; location B inferred according to system use in various locations. Students can add locations. These can be descriptive rather than physical, e.g. 'between lectures', or nonstatic locations such as 'on a train'. As with TenseITS, it is the type of location that affects a user's choice of activity. Therefore location is manually selected rather than using GPS. Once the user has selected a location, they are offered shortcuts to items they commonly use in that location, as shown in Figure 3 for a user at home, and the same user 'on the move'. Following suggestions of using 'context as a retrieval key' [13], the 'look back' button allows a user to look back to the interface of a different location if they remember accessing an item when they were there. In 'look back' mode, anything accessed contributes to the user model for the current location (not the previous location).
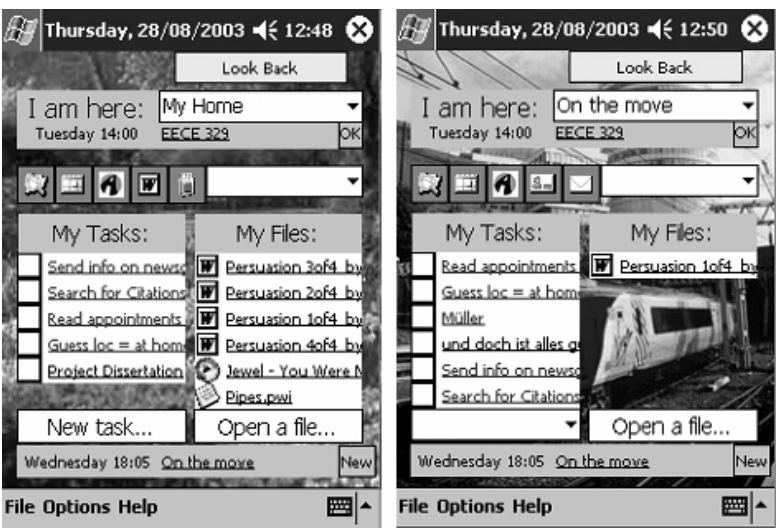

Figure 3. My Chameleon - Screens

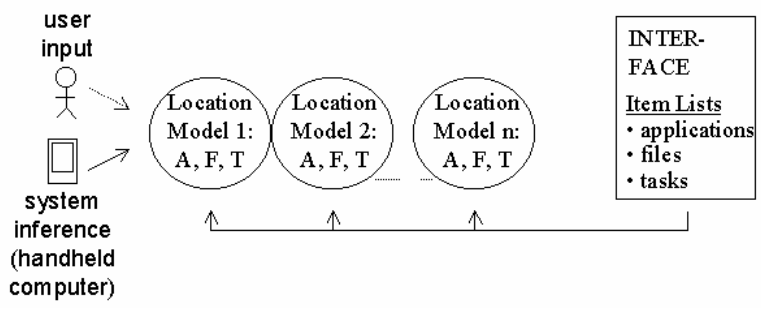

Figure 4. My Chameleon - Interaction Key: A - application; F - file; T - task

User modelling in My Chameleon is more straightforward than TenseITS (Figure 4). My Chameleon simply maintains the set of location models reflecting use or preferences in those locations. It infers appropriate links according to these preferences, taking into account information provided explicitly by the user only if the user has deleted from their user model, an item no longer required; or added a new item. Figure 3 gives two examples: at home the user has recently accessed files, applications and tasks, while on the move they have accessed mainly applications and tasks. Thus the 
first location model contains a more balanced list of tasks, applications and files, while the second contains only one file, but includes more tasks and applications.

\section{The Potential for Adaptation According to Location}

We present two small-scale exploratory studies to investigate the potential of adaptive environments for university students. Will they use them in practice? The purpose of the first study (TenseITS) was to investigate the feasibility of an approach to supporting the mobile learner that takes location-related features into account when adapting to an individual's learning needs, before developing a more complex environment to be used over longer periods. The system contained sufficient information for users to interact a few times. Thus the evaluation took at most, a few sessions. The second investigation (My Chameleon) sought to discover whether students would use an adaptive interface as a replacement for the standard mobile desktop. Will ease of access to commonly used files, applications and tasks outweigh the familiarity of the standard desktop? These investigations are only a first step, but given the increase in mobile environments being created, there is a need for more attention to be paid to features that can best facilitate learning in a mobile context. These results provide a starting point for future work.

Participants, Materials and Method: Since it is not possible to observe users throughout the day without disrupting their routine, a controlled study was not attempted. (Both systems are designed to fit into the user's normal routine.) Eight Chinese MSc students took part in the first study. The second used six (nonChinese) MSc students. In each case participants were given the software and allowed to use it as they wished, over a few days. Questionnaires were then administered with responses on a five point scale: strongly agree, agree, unsure, disagree, strongly disagree. Here we focus on just a few of the questions, in particular: whether the systems fitted naturally into users' daily routines.

Results and Discussion: Our first question is whether students will use an ITS combining learner and location/context modelling on a handheld computer, and whether such an approach is a useful learning support. Users found TenseITS suited their mobile learning needs. They considered recommendations based on their learner model (knowledge, difficulties, misconceptions) helpful, as well as recommendations taking features of their location into account, that may affect their ability to learn. Of particular interest is students' statements of use for short periods of time between other activities. 7 of the 8 students answered positively (4 strongly agreeing; 3 agreeing). More surprising is that 7 students agreed that they would use
TenseITS for up to and over an hour. This is interesting because TenseITS aims to make effective use of those short periods between activities where users may not be doing anything productive, or may be learning, but cannot take advantage of individualised sessions. Given this result, the design should be revisited to better facilitate longer use.

My Chameleon is used to access information, applications, etc. - i.e. it is intended for brief but frequent use. The My Chameleon results were also generally positive. 5 of the 6 students found the adaptive interface helpful to quickly access items (3 strongly agreeing; 2 agreeing). Most useful were the adaptive application icons and file list. Not all users used tasks, but of the 4 who did, 3 found the adaptive task list useful. The following comments were offered to an open question about why students would/would not continue using My Chameleon.

Positive: screen remembers preferences and oftenused documents next time, when in the same place; no need to find applications/files again; adds another level of organisation; much less navigation; quite convenient and useful for a student, because student activities are frequently repeated; I don't need to remember what I was doing next time, because the screen reminds me!

Negative: bit cluttered; does not conform to standard screen.

Although My Chameleon is a simple approach, it does seem that it can have an impact. Providing an option for the user to make it more closely resemble the standard screen would address the concerns of the student who commented on this difference.

We suggest that for this population of students (who are quite computer literate), the research is worth pursuing. The findings may generalise to other students who have sufficient computer familiarity. TenseITS may be useable by students with other native languages; or it might be suitable for learning other languages or non-language domains. However, because of cultural differences and differing language learning strategies of different groups [14], this needs to be tested. My Chameleon will only be used if students use computers generally, as it accesses information outside the system. As these studies were carried out with a small number of users, with the intention of discovering whether larger-scale work would be worthwhile, these and similar studies should now be extended.

Future work with TenseITS should consider a finer breakdown of time a user has available. This applies particularly in the time category 30-60 minutes, where an interaction appropriate for 30 minutes could be very different from one for 50 minutes. This is especially important if learners frequently use the system for longer periods. With a finer breakdown of time options, investigation of the ability of a learner to predict 
precisely how long they have available for this kind of interaction, will be important.

A potential problem in a full ITS is local storage on the handheld computer. It is proposed that data be stored on a desktop PC, and the learner model transferred between devices during synchronisation. All potentially relevant materials will be transferred to the handheld computer during synchronisation (see [15] for an example). These can then be automatically deleted from the handheld computer when the learner model indicates that they are no longer likely to be required.

Further work with My Chameleon should consider different screen layouts, and the possibility of allowing the user to personalise the look of their adaptive interface (the links would still adapt automatically, but users could specify the format of the interface).

Currently, each location in My Chameleon has a single model, so if a user undertakes different activities in a location on different days (e.g. preparation for different lectures), they must define the location as two locations (e.g. 'library-Mon'; 'library-Tues'), to avoid their differing behaviour being combined in a single model. Conceptually it would be neater if users could create sub-models for a single location, if required.

Different lengths of list could be useful - e.g. a learner who accesses more files than tasks might benefit if their file list was extended into the task list area.

\section{Summary}

We have considered two uses of adaptive systems in university education, that take into account features of the location that may affect choice of activity or ability to successfully complete an activity: (1) an adaptive learning environment that models understanding and location of use; (2) adaptive access to static items - files, applications and tasks, according to location.

TenseITS considers some of the key contextual features that may affect the mobile learner's ability to learn, that are often less relevant in the desktop PC situation (amount of time available for study - which may be brief; level of concentration in each location of use; likelihood of interruption by others in those locations). The system adapts the educational interaction not only to the learner's knowledge and misconceptions as in a standard ITS, but also to these additional contextual features (which, of course, may be different for different people).

My Chameleon reflects a major difference between accessing information on a desktop PC and mobile device. Desktop PCs do not usually change their location. Even if a student uses more than one PC, or has a laptop, these may be used in similar types of location. A handheld computer, however, can very easily be used when the learner is on a short bus journey; waiting for friends; on a break between lectures, etc. Because users under- take different activities with their handheld device, in different locations [7], My Chameleon was designed to facilitate access to the right items in the right place.

Initial small-scale studies suggest that learners may find adaptive mobile environments useful, and further work is recommended. A particularly interesting finding was that most students would use TenseITS for extended periods, as well as the short periods for which it was designed. Thus they are not only regarding the handheld device as a supplement to a desktop PC, when the handheld is more convenient, but in some cases they may be using it as a replacement. This assumption is supported by the logbook study [7], where some students reported using their handheld computers in the labs, even though networked PCs were available (i.e. they were not all occupied).

\section{References}

[1] Dey, A. \& Abowd, G. (1999). Towards a Better Understanding of Context and Context-Awareness, GIT-GVU-9922, College of Computing, Georgia Institute of Technology. [2] Zancanaro, M., Stock, O. \& Alfaro, I. (2003). Mobile Cinematic Presentations in a Museum Guide, MLEARN 2003: Book of Abstracts, LSDA, London, 76-77.

[3] Zimmerman, A., Lorenz, A. \& Specht, M. (2003). User Modeling in Adaptive Audio-Augmented Museum Environments, User Modeling (Proc.), Springer, Berlin, 403-407.

[4] Lonsdale, P., Baber, C., Sharples, M. (2004). A Context Awareness Architecture for Facilitating Mobile Learning, Learning with Mobile Devices (MLearn03), LSDA, 79-85.

[5] Ketamo, H. (2002). MLearning for Kindergarten's Mathematics Teaching, WMTE, IEEE Comp. Soc., 167-168.

[6] Bull, S. \& McEvoy, A.T. (2003). An Intelligent Learning Environment with an Open Learner Model for the Desktop PC and Pocket PC, Artificial Intelligence in Education (Proc.), IOS Press, Amsterdam, 389-391.

[7] Bull, S. (2003). User Modelling and Mobile Learning, User Modeling: $9^{\text {th }}$ Int. Conf., Springer, Berlin, 383-387.

[8] Sharples, M. (2000). The Design of Personal Mobile Technologies for Lifelong Learning, Computers and Education 34, 177-193.

[9] Becking, D., Betermieux, S., Bomsdorf, B., Feldmann, B., Heuel, E., Langer, P. \& Schlageter, G. (2004). Didactic Profiling: Supporting the Mobile Learner, World Conf. ELearning in Corporate, Government, Health and HE, AACE. [10] Cui, Y. \& Bull, S. (2005). Context and Learner Modelling for the Mobile Foreign Language Learner, System 33(2). [11] Chang, J. (1987). Chinese Speakers, in M. Swan \& B. Smith (eds), Learner English, CUP, Cambridge, 224-237.

[12] Schmidt, R. (1990). The Role of Consciousness in Second Language Learning, Applied Linguistics 11(2), 129-158.

[13] Lamming, M. \& Flynn, M. (1994). 'Forget-me-not' Intimate Computing in Support of Human Memory, Proc. Symposium Next Generation Human Interface, Meguro Gajoen.

[14] Oxford, R.L. (1996). (ed). Language Learning Strategies Around the World, University of Hawaii.

[15] Bull, S. \& Reid, E. (2004). Individualised Revision Material for Use on a Handheld Computer, Learning with Mobile Devices (MLearn03), LSDA, 35-42. 